\title{
Brolucizumab vs aflibercept and ranibizumab for neovascular age-related macular degeneration: a cost-effectiveness analysis
}

\author{
Justin S Yu, PharmD, MS; Rashad Carlton, PharmD, MSPH; Neetu Agashivala, BPharm, MS; Tarek Hassan, MD; \\ and Charles C Wykoff, MD, PhD
}

\section{What is already known about this subject}

- Neovascular (wet) age-related macular degeneration (AMD) is a devastating eye disease and the leading cause of blindness worldwide.

- Unmet needs still remain in treating neovascular AMD because of treatment burden and suboptimal treatment response to current antivascular endothelial growth factor (anti-VEGF) agents.

\section{What this study adds}

- This is the first published costeffectiveness model that compares brolucizumab to other anti-VEGF agents in the treatment of wet AMD.

- The results of this model demonstrate that, according to phase 3 protocolguided dosing regimens, brolucizumab is a cost-effective treatment for wet AMD.

\begin{abstract}
Author affiliations
Justin S Yu, PharmD, MS, and Neetu Agashivala, BPharm, MS, Novartis Pharmaceuticals Corporation, East Hanover, NJ. Rashad Carlton, PharmD, MSPH, Xcenda LLC, Palm Harbor, FL; Tarek Hassan, MD, Oakland University William Beaumont School of Medicine and Associated Retinal Consultants, Royal Oak, Ml; and Charles C Wykoff, MD, PhD, Retina Consultants of Houston, Retina Consultants of America, and Blanton Eye Institute and Houston Methodist Hospital, Houston, TX.
\end{abstract}

AUTHOR CORRESPONDENCE:

Neetu Agashivala, 862.778.8300;

neetu.agashivala@novartis.com

$J$ Manag Care Spec Pharm 2021;27(6):743-52

Copyright $\odot 2021$, Academy of Managed Care Pharmacy. All rights reserved.

AMD, attempts to mitigate treatment burden through less frequent injections.

OBJECTIVE: To assess the incremental costeffectiveness of brolucizumab compared with aflibercept and ranibizumab, given similar costs per injection and the potential for longer dosing intervals based on phase 3 clinical trial data.

METHODS: A Markov model was developed to model the treatment of wet AMD patients with brolucizumab vs aflibercept and vs ranibizumab over a lifetime time horizon (base case) and 5-year time horizon (scenario analysis). The Markov model consisted of 3 primary health states: on treatment, off treatment, and death. Markov substates (5 total) described visual acuity (VA) ranging from no vision impairment to blindness. These VA-based substates were defined by best-corrected visual acuity (BCVA) values measured using Early Treatment Diabetic Retinopathy Study letters. Fixed-dosing regimens for each therapy were included in the model: dosing every 4 weeks ( $q 4 w$ ) for the first 3 months followed by dosing q8w/q12w 
for brolucizumab, dosing $q 4 \mathrm{w}$ for the first 3 months followed by dosing $q 8 w$ for aflibercept, and $q 4 \mathrm{w}$ for ranibizumab.

RESULTS: In the base case, brolucizumab was less costly than aflibercept $(\$ 63,614$ vs $\$ 72,189)$, and brolucizumab generated 0.0079 more quality-adjusted life-years (QALYs) than aflibercept (4.580 vs 4.572). Lower total costs with brolucizumab were driven by reduced drug costs $(\$ 56,432$ vs $\$ 64,057)$, reduced administration costs $(\$ 6,013$ vs $\$ 6,825)$, and reduced monitoring costs ( $\$ 1,168$ vs $\$ 1,306)$. When evaluating the cost-effectiveness of brolucizumab over a 5 -year time horizon, brolucizumab was less costly than aflibercept $(\$ 44,644$ vs $\$ 50,772)$ and generated an additional 0.0049 QALYs (2.953 vs 2.948). Additionally, brolucizumab was less costly than ranibizumab $(\$ 63,614$ vs $\$ 128,163$ ) and generated 0.0078 more QALYs than ranibizumab (4.580 vs 4.572 ) in the base case. Lower total costs with brolucizumab were driven by reduced drug costs $(\$ 56,432$ vs $\$ 114,516)$, reduced administration costs ( $\$ 6,013$ vs $\$ 11,541)$, and reduced monitoring costs $(\$ 1,168$ vs $\$ 2,107)$. When evaluating the cost-effectiveness of brolucizumab over a 5 -year time horizon, brolucizumab was less costly than ranibizumab ( $\$ 44,644$ vs $\$ 89,665)$, and brolucizumab generated an additional 0.0046 QALYs (2.953 vs 2.948).

CONCLUSIONS: Brolucizumab can be cost saving and cost-effective compared with aflibercept and ranibizumab in the treatment of wet AMD.

Age-related macular degeneration (AMD) is a leading cause of blindness worldwide and is the most common cause of blindness in developed countries., ${ }^{1,2}$ Advanced AMD can be dichotomized into 2 primary clinical/pathologic classifications: non-neovascular (ie, "dry" or atrophic) AMD and neovascular (ie, "wet" or exudative) AMD. Early stages and atrophic AMD comprise approximately $85 \%-90 \%$ of all cases of AMD, yet they are associated with lower disease burden. Wet AMD, in contrast, is more severe, despite only accounting for $10 \%-15 \%$ of cases of AMD. ${ }^{3}$ While wet AMD is the less common form of AMD, it has historically accounted for $80 \%-90 \%$ of cases of associated legal blindness. ${ }^{1,3-7}$

The economic burden of wet AMD is substantial. One US-based survey $(\mathrm{N}=200)$ found decreasing vision secondary to wet AMD to be positively correlated with increasing societal costs $(\mathrm{P}<0.001)$, including direct medical costs, direct nonmedical costs, and indirect costs, such as those due to loss of employment and lower salary. ${ }^{8}$ Specifically, mean annual costs were found to be $\$ 39,910$ per wet AMD patient compared with $\$ 6,116$ per nonwet AMD control patient $(P<0.001)$. Similarly, it has been demonstrated that incremental loss of visual acuity (VA) is associated with increasing costs. ${ }^{8}$ Another study estimated that the annual cost of low vision alone in the United States is $\$ 15,900$ per person in individuals with vision loss and $\$ 26,900$ per person in individuals with blindness. Among these costs, direct costs comprised approximately $50 \%$ of total costs. ${ }^{9,10}$

From a quality-of-life perspective, wet AMD has been associated with a $13 \%$ reduction in well-being, as assessed by the EuroQoL-5D (wet AMD patients: 0.95 [95\% CI = 0.600.69] vs non-AMD control patients: 0.75 [95\% $\mathrm{CI}=0.71-0.79]$, $\mathrm{P}<0.001)$ in addition to a $30 \%$ increase in anxiety, $42 \%$ increase in depression symptoms, and $45 \%$ worse visionrelated functioning after adjusting for covariates $(\mathrm{P}<0.001$ for each). ${ }^{11}$ These detriments to quality of life were compounded by observation of a doubling in falls (16\% in wet AMD vs $8 \%$ in non-AMD controls, $\mathrm{P}<0.001)$, as well as a quadrupling of patients who needed assistance in order to perform activities of daily living (29\% vs $7 \%$ in wet AMD and non-AMD controls, respectively, $\mathrm{P}<0.001)$.

\section{OUTCOMES RESEARCH IN WET AMD}

Given the causal relationship between wet AMD and blindness, as well as the progressive nature of wet AMD, it is unsurprising that VA is a clinically significant outcome of interest in the study of wet AMD and in studies of corresponding interventions aimed at preventing or delaying disease progression. Given its clinical significance, VA change from baseline, as measured in terms of bestcorrected visual acuity (BCVA), is frequently used as a primary endpoint in interventional clinical trials in wet AMD. ${ }^{12,13}$

While measures of patient-reported VA are well-accepted clinical outcomes, research on wet AMD is progressing and developing beyond that of BCVA toward more nuanced clinician-reported and objective outcomes that can provide additional insights into disease burden, disease progression, and interventional efficacy. ${ }^{14,15}$ To this end, emerging research suggests that controlling retinal fluid accumulation is a key factor for maintaining and improving vision. ${ }^{16}$ Retinal fluid accumulation, for example, is considered one of the leading diagnostic criteria for wet AMD. ${ }^{17,18}$ Further, according to a 2018 survey of 1,028 retinal specialists, retinal fluid accumulation is one of the most important indicators and predictors of disease activity. ${ }^{19}$

\section{TREATMENTS FOR WET AMD}

Antivascular endothelial growth factor-A (anti-VEGF) therapy delivered via intravitreal injection into the eyes of patients with wet AMD is well established as a standard of care treatment for the disease. ${ }^{12,20-23}$ Despite demonstrating improvements in visual and anatomical outcomes, ${ }^{12,20-23}$ unmet needs remain, particularly those related to the substantial treatment burden associated with the currently available anti-VEGF therapies. A survey of retina specialists, for example, demonstrated that the greatest unmet 


\section{FIGURE 1 Markov Model Primary Health States and BCVA-Based Markov Substates}

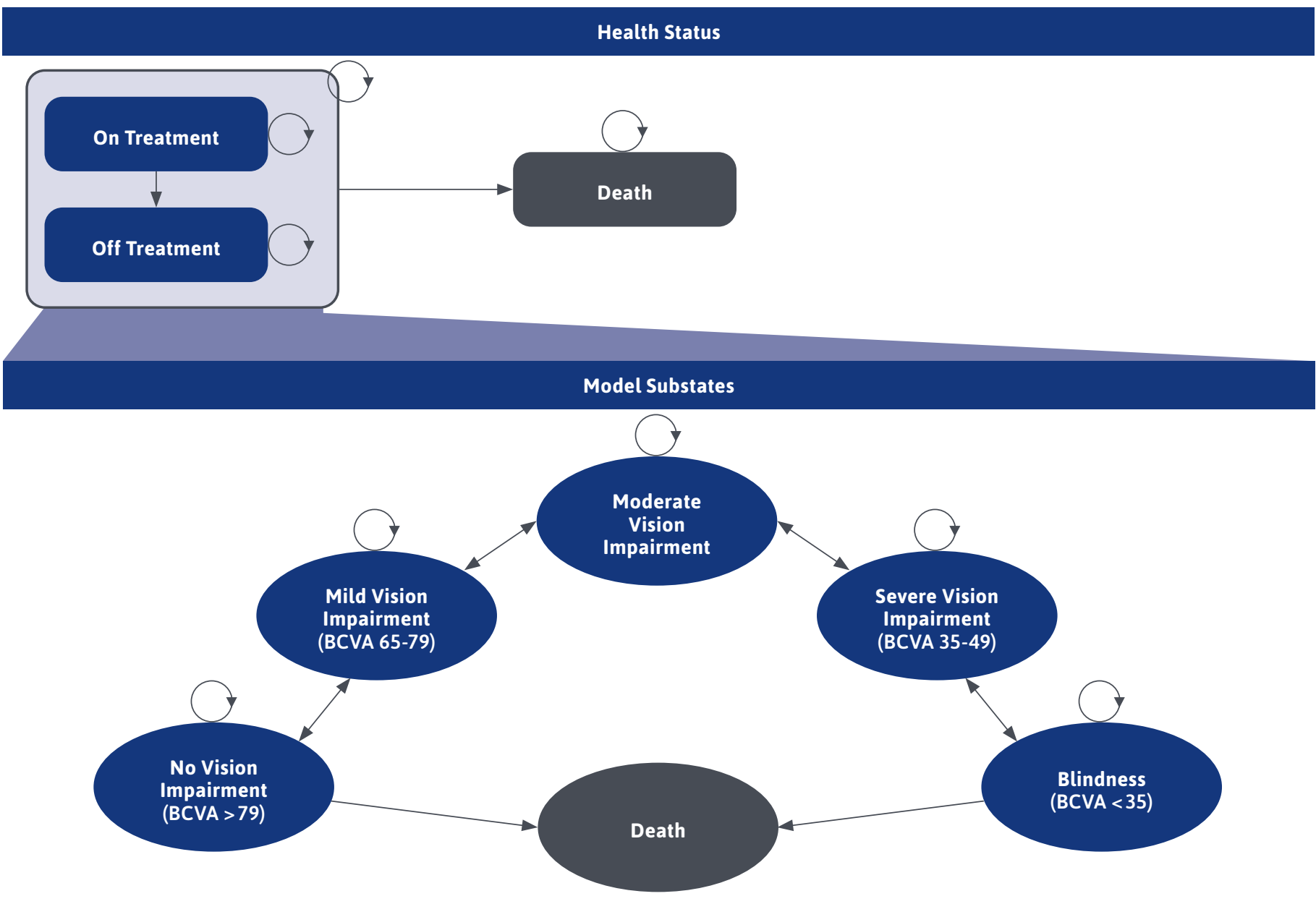

$B C V A=$ best-corrected visual acuity (measured using the Early Treatment Diabetic Retinopathy Study letters score).

needs regarding wet AMD treatment were reduced treatment burden and long-acting/sustained delivery. ${ }^{19}$ Current treatments require frequent administration, with dosing intervals as short as 4 weeks, as described by US Food and Drug Administration (FDA) prescribing information. Specifically, 2 well-established anti-VEGF therapies, aflibercept and ranibizumab, were initially studied and approved based on dosing regimens of once every 4 weeks or once every 8 weeks after 3 monthly doses. ${ }^{24,25}$ Given this administration-related burden, adherence to and persistence on long-term therapy remain a challenge. Dosing intervals tend to be less frequent in clinical practice, as treatment adherence tends to be harder to maintain compared with the clinical trial settings. ${ }^{26}$ Corresponding difficulties in achieving long-term visual outcomes have also been documented. ${ }^{27-29}$

Brolucizumab-dbll (ie, brolucizumab) is a first-in-kind, humanized single-chain antibody fragment ( $\mathrm{scFv}$ ) vascular endothelial growth factor A inhibitor approved by the FDA for the treatment of patients with wet AMD. ${ }^{30}$ The efficacy and safety of brolucizumab have been established in the HAWK and HARRIER clinical trials, which were 2 separate, 2-year, randomized, double-masked, multicenter, activecontrolled trials that measured the comparative safety and efficacy of brolucizumab and aflibercept in the treatment of patients with wet AMD..$^{15}$ In HAWK and HARRIER, at week 48 , brolucizumab was non-inferior to aflibercept in BCVA change from baseline: +6.6 letters for brolucizumab 
vs +6.8 letters for aflibercept in HAWK, and +6.9 letters for brolucizumab vs +7.6 letters for aflibercept in HARRIER.

Given the clinical efficacy, safety, and dosing profiles of brolucizumab, aflibercept, and ranibizumab, the comparative effectiveness and costs of these treatments represent key factors in determining their value relative to one another in the context of limited health care resources. The objective of this study was therefore to assess the incremental cost-effectiveness of brolucizumab compared with aflibercept and ranibizumab from a US payer perspective, using phase 3 , protocol-guided retreatment schedules, in light of their similar cost per injection and brolucizumab's potential for longer dosing intervals.

This study was exempt from approval by an institutional review board, since it did not use or collect human subject research or identifiable private information. All datasets used in the study were deidentified by an independent third party before the authors' initial review. This study did not assess a treatment effect using real-world evidence. This study evaluated published clinical and economic data in the development of a cost-effectiveness model.

\section{Methods}

\section{MODEL STRUCTURE}

A Markov model was developed in Microsoft Excel to model the treatment of wet AMD patients with brolucizumab compared with aflibercept and ranibizumab over a lifetime time horizon (base case) and 5-year time horizon (scenario analysis) from a US payer perspective.

The Markov model consisted of 3 primary health states: on treatment, off treatment, and death. These health states were defined in accordance with single-eye wet AMD involvement. VA in the unaffected eye (ie, the fellow eye) was assumed to remain stable.

Markov substates were also incorporated into the model; these substates described the extent of visual impairment and ranged from no vision impairment to blindness, as well as death. These VA-based substates were defined by BCVA values measured using Early Treatment Diabetic Retinopathy Study letters, ranging from more than 79 to less than 35 for no visual impairment and blindness, respectively. Figure 1 depicts the Markov model primary health states and BCVA-based Markov substates.

During each model cycle of 1 year, patients could remain at the current level of BCVA, improve by at least 15 letters (1 health state), worsen by at least 15 letters ( 1 health state), or transition to death. Patients in the no vision impairment health state could remain in the current health state, worsen by at least 15 letters ( 1 health state), or transition to death. Additionally, patients in the blindness health state could remain in the current health state, improve by at least 15 letters (1 health state), or transition to death.

\section{GENERAL INPUTS}

In the base case, outcomes and costs were discounted at a rate of $3 \%$ annually. The target patient population consisted of patients with wet AMD, with a default cohort starting age of 76 years. Baseline mortality was based on the age of the cohort in question, as well as on the increased risk of mortality due to AMD. Specifically, a relative risk of mortality secondary to AMD of 1.09 was incorporated in the model in order to inform mortality rates in AMD compared with unadjusted levels. ${ }^{31}$

Treatment was assumed to be provided for a lifetime unless otherwise stated; anti-VEGF therapeutic switching is not accounted for within the model. Initial disease severity rates for both treated and fellow eyes across treatment cohorts were derived from a pooled analysis of HAWK and HARRIER trials. ${ }^{15}$ See Supplementary Table 1 in Appendix 1 (available in online article) for an overview of key model inputs.

\section{COST INPUTS}

Costs in the model solely comprise direct medical costs and were inclusive of drug costs, interventional administration costs (ie, intravitreal injection costs, office visit costs), and monitoring costs. Costs per anti-VEGF dose (ie, drug costs) were derived from IBM Micromedex RED BOOK using wholesale acquisition costs; costs of intravitreal injection and office visit costs were taken from the corresponding Current Procedural Terminology (CPT) codes in the Centers for Medicare \& Medicaid Services Physician Fee Schedule. ${ }^{32,33}$ Monitoring and disease-related costs included the cost of optical coherence tomography (OCT), the cost of fluorescein angiography, and a multiplier for bilateral fluorescein angiography (in the event that both eyes were tested). These values were derived from corresponding CPT codes and available published data..$^{9,33}$ Additionally, all patients were assumed to have received an OCT at each treatment visit. A fluorescein angiography was assumed to have been performed only at the initial visit. ${ }^{12}$

\section{DOSING}

Brolucizumab was dosed and administered in accordance with the regimens studied in the phase 3 HAWK and HARRIER clinical trials. ${ }^{15}$ The lowest-frequency fixed-dosing regimens considered to be most effective based on product prescribing information were included in the model: every 8 weeks (q8w)/q12w for brolucizumab, q8w for aflibercept, 


\section{FIGURE 2 OWSA Results (ICER Brolucizumab vs Aflibercept)}

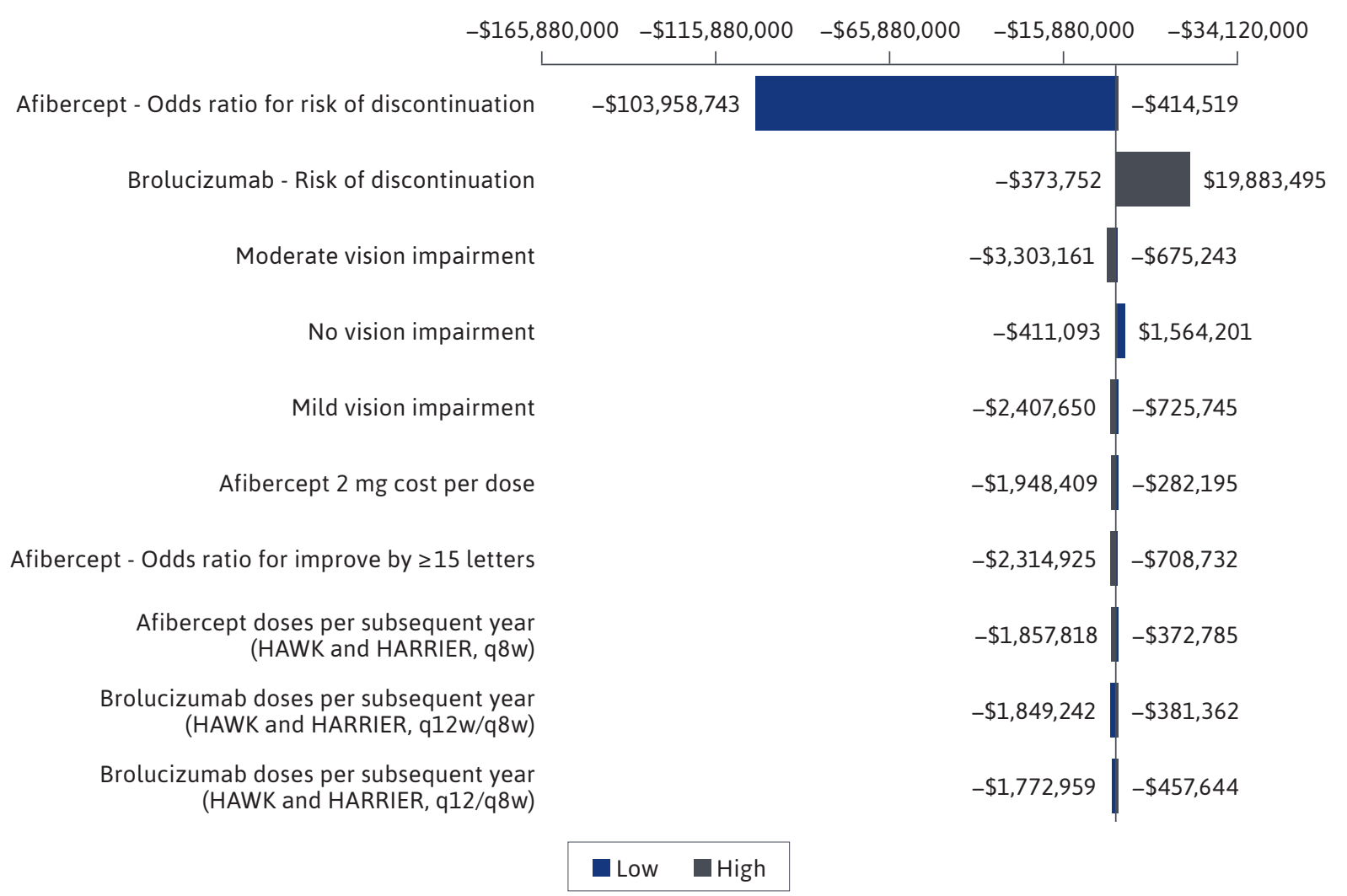

$I C E R=$ incremental cost-effectiveness ratio; OWSA = one-way sensitivity analysis; $q 8 \mathrm{w}=$ once every 8 weeks; qw12 = once every 12 weeks.

and q4w for ranibizumab. ${ }^{24,25,30}$ In an attempt to mirror realworld dosing paradigms, brolucizumab, aflibercept, and ranibizumab dosing inputs were stratified into those that corresponded to initial annual dose quantities (inclusive of loading doses) and subsequent annual dose quantities; note that real-world dosing and monitoring frequencies and requirements for each anti-VEGF agent may vary based on individual patient needs. The risk of treatment discontinuation was assumed to be equal among all treatments.

\section{EFFICACY INPUTS}

BCVA improvement and worsening inputs for brolucizumab were derived from a pooled analysis of the head-tohead HAWK and HARRIER trials. ${ }^{15}$ The efficacy inputs for aflibercept and ranibizumab were based on the results of a systematic literature review and network meta-analysis (NMA) of randomized controlled clinical trials assessing the efficacy and safety of treatments for wet AMD. Odds ratios describing relative improvement or worsening by at least 15 letters were derived for aflibercept and ranibizumab vs brolucizumab in the NMA. ${ }^{15,21,22,34-46}$ See Supplementary Appendix 2 (available in online article) for key results from the NMA.

The aflibercept and ranibizumab odds ratios were applied to the baseline probabilities of improving and worsening by at least 15 letters for brolucizumab in order to derive aflibercept and ranibizumab efficacy values. The probability of improving by at least 15 letters was $31.23 \%$ for brolucizumab, 27.61\% for aflibercept, and 29.01\% for ranibizumab. ${ }^{15}$ The probability of worsening by at least 15 letters was $5.07 \%$ for brolucizumab, $5.21 \%$ for aflibercept, and $6.21 \%$ for ranibizumab. ${ }^{15}$ In the absence of treatment, and thus while within the model's off-treatment state, disease progression expressed by the loss of at least 15 letters in BCVA was set at an annual rate of $37.80 \%$ in the base case. 


\section{FIGURE 3 PSA Scatterplot (Brolucizumab vs Aflibercept)}

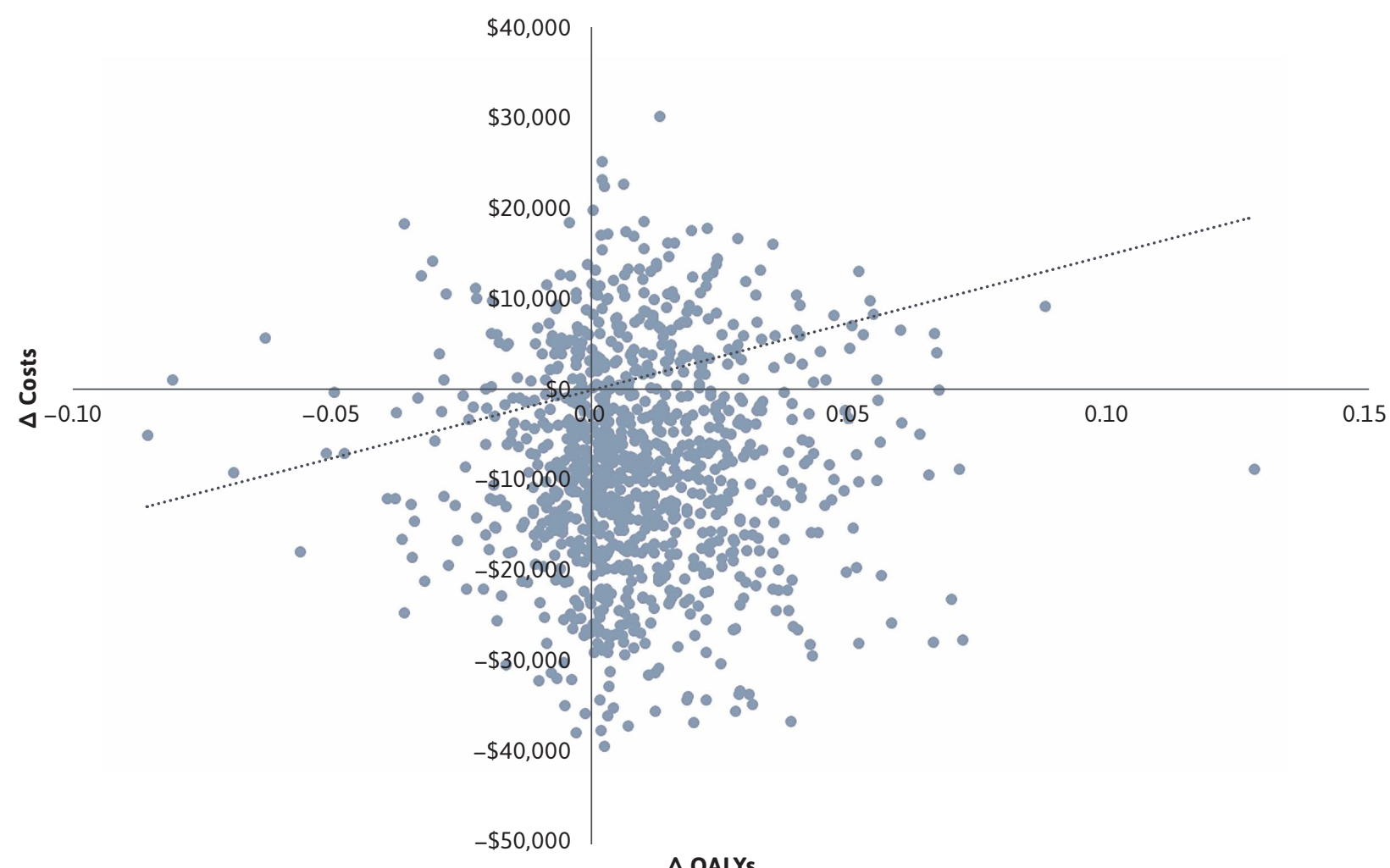

Notes: Willingness to pay $=\$ 150,000$ per QALY; incremental costs = total costs brolucizumab - total costs aflibercept; incremental QALYs = total QALYs brolucizumab - total QALYs aflibercept.

$P S A=$ probabilistic sensitivity analysis; $Q A L Y=$ quality-adjusted life-year.

\section{UTILITY INPUTS}

Utility inputs for each BCVA-linked Markov state were derived from Brown et $\mathrm{al}_{,}{ }^{47}$ which used a time trade-off methodology in order to derive utility values for each state. Utilities ranged from 0.78 to 0.88 for blindness and no vision impairment, respectively. A utility decrement of 0.1 was assumed between the best and worst BCVA substates for the treated eye. ${ }^{48}$

\section{MODEL OUTPUT}

The output of the model was defined in terms of incremental cost per quality-adjusted life-year (QALY) gained (ie, the incremental cost-effectiveness ratio [ICER]) of brolucizumab vs aflibercept and vs ranibizumab. Total QALYs were calculated as the sum of the utility values for each level of BCVA Markov state multiplied by the percentage of time that patients remained in the health state.

\section{SENSITIVITY ANALYSIS}

A one-way deterministic sensitivity analysis (OWSA) was conducted in addition to a multivariate probabilistic sensitivity analysis (PSA) for the base case. Parameters in the OWSA included discount rates, relative risk for mortality, improvement/worsening by 15 letters for each treatment, utility inputs, rates of discontinuation, dosing schedules, and costs. Parameter values for the PSA were defined in accordance with the appropriate statistical distribution for each variable type (ie, beta, Dirichlet, gamma, log normal; Supplementary Figure 1, available in online article). For the OWSA, a tornado diagram illustrating which model parameters and inputs the model and its ICER outputs were most sensitive to was developed (Figure 2 and Supplementary Figure 2, available in online article). For the PSA, 1,000 simulations were run, and the results were presented on a cost-effectiveness scatterplot (Figure 3 and 


\begin{tabular}{l|c|c|c}
\hline \multicolumn{1}{c}{ TABLE 1} & Base-Case Model Results \\
\hline Costs & Brolucizumab & Aflibercept & Ranibizumab \\
\hline Drug costs & $\$ 56,432$ & $\$ 64,057$ & $\$ 114,516$ \\
\hline Administration costs & $\$ 6,013$ & $\$ 6,825$ & $\$ 11,541$ \\
\hline Monitoring costs & $\$ 1,168$ & $\$ 1,306$ & $\$ 2,107$ \\
\hline Total costs & $\$ 63,614$ & $\$ 72,189$ & $\$ 128,163$ \\
\hline Outcomes & 4.580 & 4.572 & 4.572 \\
\hline QALYs & & \\
\hline QALY=quality-adjusted life-year.
\end{tabular}

Supplementary Figure 2, available in online article). Additionally, for the PSA, a cost-effectiveness acceptability curve was developed using the Institute for Clinical and Economic Review's value-based willingness-to-pay benchmark of $\$ 150,000$ per QALY. ${ }^{49}$

\section{Results}

\section{BASE-CASE RESULTS}

In the model base case with a lifetime horizon, brolucizumab was less costly than aflibercept $(\$ 63,614$ vs $\$ 72,189)$. Lower total costs with brolucizumab were driven by reduced drug costs (\$56,432 vs \$64,057), reduced administration costs (\$6,013 vs $\$ 6,825)$, and reduced monitoring costs (\$1,168 vs $\$ 1,306)$. Brolucizumab generated an additional 0.0077 QALYs compared with aflibercept (4.580 vs 4.572$)$. Since brolucizumab was less costly and more effective than aflibercept, an ICER value was not calculated, as brolucizumab was a dominant treatment over aflibercept.

Additionally, brolucizumab was less costly than ranibizumab (\$63,614 vs $\$ 128,163)$. Lower total costs with brolucizumab were driven by reduced drug costs $(\$ 56,432$ vs $\$ 114,516)$, reduced administration costs $(\$ 6,013$ vs $\$ 11,541)$, and reduced monitoring costs (\$1,168 vs $\$ 2,107)$. Brolucizumab generated an additional 0.0078 QALYs compared with ranibizumab (4.580 vs 4.572). Since brolucizumab was less costly and more effective than ranibizumab, an ICER value was not calculated, as brolucizumab was a dominant treatment over ranibizumab. Table 1 describes the base-case model results.

\section{FIVE-YEAR TIME HORIZON RESULTS}

When evaluating the cost-effectiveness over a 5-year time horizon, brolucizumab was less costly than aflibercept $(\$ 44,644$ vs $\$ 50,772)$. Lower total costs with brolucizumab

\begin{tabular}{|c|c|c|c|}
\hline Parameter & Brolucizumab & Aflibercept & Ranibizumab \\
\hline \multicolumn{4}{|l|}{ Costs } \\
\hline Drug costs & $\$ 39,389$ & $\$ 44,815$ & $\$ 79,745$ \\
\hline Administration costs & $\$ 4,359$ & $\$ 4,958$ & $\$ 8,339$ \\
\hline Monitoring costs & $\$ 896$ & $\$ 1,000$ & $\$ 1,581$ \\
\hline Total costs & $\$ 44,644$ & $\$ 50,772$ & $\$ 89,665$ \\
\hline \multicolumn{4}{|l|}{ Outcomes } \\
\hline QALYS & 2.953 & 2.948 & 2.948 \\
\hline
\end{tabular}

were driven by reduced drug costs $(\$ 39,389$ vs $\$ 44,815)$, reduced administration costs $(\$ 4,359$ vs $\$ 4,958)$, and reduced monitoring costs (\$896 vs $\$ 1,000)$. Brolucizumab generated an additional 0.0049 QALYs compared with aflibercept (2.953 vs 2.948). When evaluating the costeffectiveness of brolucizumab over a 5-year time horizon, brolucizumab was less costly than ranibizumab (\$44,644 vs $\$ 89,665)$ and generated an additional 0.0046 QALYs compared with ranibizumab (2.953 vs 2.948 ). Brolucizumab was a dominant treatment compared with aflibercept over a 5 -year time horizon. Table 2 describes the model results for a 5-year time horizon.

\section{ONE-WAY SENSITIVITY ANALYSIS RESULTS}

In the OWSA results, key model parameters were varied by $\pm 10 \%$. The variables with the biggest impact on the model for brolucizumab vs aflibercept were the odds ratio for discontinuation of aflibercept, the risk of discontinuation of brolucizumab, and the baseline population disease severity distribution value for moderate vision impairment as determined by BCVA letters. The variables with the biggest impact on the model for brolucizumab vs ranibizumab were the odds ratio for the risk of discontinuation of ranibizumab, the risk of discontinuation of brolucizumab, and the baseline population disease severity distribution value for no vision impairment. Figure 2 depicts base-case OWSA results.

\section{PROBABILISTIC SENSITIVITY ANALYSIS RESULTS}

In the PSA, at a willingness to pay of $\$ 150,000$ per QALY, brolucizumab was cost-effective vs aflibercept over $76 \%$ of the time and cost-effective vs ranibizumab $100 \%$ of the time. In $76 \%$ and $100 \%$ of the simulations, brolucizumab was less costly than aflibercept and ranibizumab, respectively. In $71 \%$ and $68 \%$ of the simulations, brolucizumab was more effective than aflibercept and ranibizumab, respectively. Figure 3 depicts the PSA scatterplot results. 


\section{Discussion}

The objective of this cost-effectiveness analysis was to use available phase 3 , protocol-guided retreatment regimens to assess the cost-effectiveness of brolucizumab compared with aflibercept and ranibizumab from a US payer perspective, in light of their similar cost per injection and extended dosing intervals with brolucizumab for the treatment of wet AMD. Brolucizumab was more cost-effective than aflibercept and ranibizumab, given a short time horizon (5 years) and a lifetime time horizon.

While not approved by the FDA for ophthalmic use, intravitreal bevacizumab has been used as an off-label treatment for wet AMD. Research has shown that off-label bevacizumab has comparable efficacy to ranibizumab in VA. ${ }^{34}$ Due to lower costs of treatment, intravitreal bevacizumab has historically been used at a higher rate than approved ranibizumab for wet AMD. ${ }^{50}$ Since this current analysis focused on FDA-approved treatments for wet AMD based on phase 3 protocol-guided treatment regimens, intravitreal bevacizumab was excluded.

This is the first cost-effectiveness model of its kind to evaluate brolucizumab vs other FDA-approved anti-VEGF agents for the treatment wet AMD. Previous cost-effectiveness research has examined the cost-effectiveness of aflibercept and ranibizumab for wet AMD. One study using a similar Markov structure with on-treatment and off-treatment health states, and substates based on BCVA found that aflibercept was cost-effective compared with ranibizumab for the treatment of wet AMD. ${ }^{51}$ The findings of this study are consistent with earlier analyses showing similar QALY gains with aflibercept and ranibizumab, with lower costs for aflibercept compared with ranibizumab.

While other cost-effectiveness analyses of anti-VEGF agents have not specifically investigated general trends associated with variable dosing intervals and corresponding outcomes, some noneconomic analyses have aimed to achieve this end. Specifically, one retrospective analysis conducted by Ferreira et al found that, in a real-world setting, ranibizumab and aflibercept were associated with $4.9 \pm 3.3$ and $5.2 \pm 2.9$ injections per year, respectively. ${ }^{52}$ In comparison, our model used more frequent dosing, per prescribing information for all model comparators. Given the less frequent dosing for aflibercept and ranibizumab in the real-world setting, ${ }^{52,53}$ it is reasonable to postulate that under real-world parameters, brolucizumab would be associated with even fewer doses compared with the prescribing information and, potentially, compared with other anti-VEGF agents.

\section{LIMITATIONS}

There were several limitations to this cost-effectiveness analysis. First, the model used the phase 3, protocol-guided retreatment regimens to determine injection frequency. These regimens may not be reflective of real-world treatment for many patients with wet AMD.

Second, a Markov model evaluates cohorts of patients during each cycle, as opposed to individual patients during each cycle.

Third, the model did not include costs related to adverse events, the impact of adverse events on costeffectiveness, or the impact of adverse events on QALYs. In the real-world setting, a variety of adverse events may occur with anti-VEGF treatment, some independent of the specific anti-VEGF agent used, and some potentially related to the specific anti-VEGF agent used, as demonstrated in clinical trials.

Further, from an effectiveness perspective, other potential benefits of treatment, such as long-term reduction in retinal fluid, were not captured in the model. Because retinal fluid reduction had not been previously mapped to a utility measure, the incremental clinical and costeffectiveness across these agents in accordance with this emerging and clinically significant outcome measure cannot be fully informed. Future attempts may be considered to map retinal fluid reduction to utility values in order to better differentiate and elucidate the incremental value of anti-VEGF therapies compared with one another.

Simplified assumptions were made in the current model. First, it was assumed that the risk of improving or worsening by at least 15 letters at each year were independent of current BCVA levels. This aligns with the way that BCVA improvement/worsening was presented in the HAWK and HARRIER trials. The model also assumed that the rates of improving/worsening BCVA levels were consistent throughout the model. In the absence of long-term clinical trials, the true rate of improvement/worsening of BCVA beyond the clinical trial duration is unknown and is subject to uncertainty.

Finally, this model did not capture direct nonmedical costs or indirect costs associated with the presence of and treatment for wet AMD.

\section{Conclusions}

In this cost-effectiveness analysis, brolucizumab was less costly and more cost-effective than aflibercept and ranibizumab in the treatment of wet AMD. This is a function of brolucizumab's greater improvement by at least 15 letters, lower rate of worsening by at least 15 letters, and 
fewer initial and subsequent doses per year, as determined by the phase 3 , protocol-guided retreatment regimens. The most powerful of these drivers appears to be the number of doses per year. Future research should attempt to capture the health economic-related benefits of retinal fluid reduction associated with anti-VEGF agents in the treatment of wet AMD.

\section{DISCLOSURES}

Novartis Pharmaceuticals Corporation provided funding to Xcenda for the costeffectiveness analysis and preparation of this manuscript. Carlton is an employee of Xcenda. Agashivala is employed by Novartis Pharmaceuticals Corporation; Yu was an employee of Novartis Pharmaceutical Corporation at the time of this study.

Hassan reports personal fees from iOPEN, BVI/Visitrec, ArcticDx, Bayer, F. Hoffmann-La Roche Ltd, Broadspot, BMC, Katalyst Surgical, Alcon, Vitreq, Surgicube, personal Ocugenix, Regeneron, Allergan, Oculus Surgical, Novartis, Genentech, and Eyepoint, unrelated to this work.

Wykoff reports personal fees from Corcept Therapeutics, DORC, EyePoint, Gyroscope, IVERIC Bio, Merck, Notal Vision, ONL Therapeutics, Oxurion, Palatin, PolyPhotonix, Takeda, Thea Open Innovation; grants from Aerie Pharmaceuticals, Aldeyra, Gemini Therapeutics, Graybug Vision, IONIS Pharmaceutical, LMRI, Mylan, Neurotech Pharmaceuticals, Outlook Pharmaceuticals, Samsung Bioepis, Senju, Taiwan Liposome Company, Xbrane BioPharma, Santen; and grants and personal fees from Adverum, Allergan, Apellis, Chengdu Kanghong Biotechnologies (KHB), Clearside Biomedical, Genentech, Kodiak Sciences, NGM Biopharmaceuticals, Novartis, Opthea, Recens Medical, Regenxbio, Roche, and Regeneron, unrelated to this work.

This research was presented as a virtual poster at the AMCP 2020 Annual Meeting, April 2020.

\section{REFERENCES}

1. Bird AC. Therapeutic targets in agerelated macular disease. J Clin Invest. 2010;120(9):3033-41.
2. Wang H, Hartnett ME. Regulation of signaling events involved in the pathophysiology of neovascular AMD. Mol Vis. 2016;22:189-202.

3. Mogk LG, Duffy MA. The difference between wet and dry age-related macular degeneration. Accessed November 19, 2018. http://www.visionaware. org/info/your-eye-condition/agerelated-macular-degeneration-amd/ wet-and-dry-amd/125

4. Ba J, Peng RS, Xu D, et al. Intravitreal anti-VEGF injections for treating wet age-related macular degeneration: a systematic review and meta-analysis. Drug Des Devel Ther. 2015;9:5397-405.

5. Pennington KL, DeAngelis MM. Epidemiology of age-related macular degeneration (AMD): associations with cardiovascular disease phenotypes and lipid factors. Eye Vis (Lond). 2016;22;3:34.

6. Yonekawa Y, Miller JW, Kim IK. Agerelated macular degeneration: advances in management and diagnosis. J Clin Med. 2015;4(2):343-59.

7. Wong TY, Chakravarthy U, Klein R, et al. The natural history and prognosis of neovascular age-related macular degeneration: a systematic review of the literature and meta-analysis. Ophthalmology. 2008;115(1):116-26.

8. Brown MM, Brown GC, Lieske HB, Tran I, Turpcu A, Colman S. Societal costs associated with neovascular age-related macular degeneration in the United States. Retina. 2016;36(2):285-98.

9. Wittenborn JS, Rein D. Cost of vision problems: the economic burden of vision loss and eye disorders in the United States. 2013. Accessed May 5, 2021. http:// preventblindness.org/wp-content/ uploads/2020/04/Economic-Burden-ofVision-Final-Report_130611_0.pdf

10. NORC at the University of Chicago. The economic burden of vision loss and eye disorders in the United States. Accessed May 17, 2019. http://www. norc.org/Research/Projects/Pages/ the-economic-burden-of-vision-loss-andeye-disorders-in-the-united-states.aspx

11. Soubrane G, Cruess A, Lotery A, et al. Burden and health care resource utilization in neovascular age-related macular degeneration: findings of a multicountry study. Arch Ophthalmol. 2007;125(9):1249-54.

12. American Academy of Ophthalmology. Age-Related Macular Degeneration Preferred Practice Pattern $®$ Guidelines. Elsevier; 2019. Accessed August 3, 2020. https://www.aaojournal.org/article/ $\underline{\text { S0161-6420(19)32091-3/pdf }}$
13. Mehta H, Tufail A, Daien V, et al. Real-world outcomes in patients with neovascular age-related macular degeneration treated with intravitreal vascular endothelial growth factor inhibitors. Prog Retin Eye Res. 2018;65:127-46.

14. Wykoff C, Clark W, Nielsen J, et al. Optimizing anti-VEGF treatment outcomes for patients with neovascular age-related macular degeneration. J Manag Care Spec Pharm. 2018;24(2-a Suppl):S3-S15. doi: 10.18553/ jmcp.2018.24.2-a.s3

15. Dugel PU, Koh A, Ogura Y, et al. HAWK and HARRIER: phase 3, multicenter, randomized, double-masked trials of brolucizumab-dbll for neovascular age-related macular degeneration. Ophthalmology. 2020;127(1):72-84. doi: 10.1016/j.ophtha.2019.04.017

16. Spaide R. Ranibizumab according to need: a treatment for age-related macular degeneration. Am J Ophthalmol. 2007;143(4):679-80.

17. Arnold JJ, Markey CM, Kurstjens NP, Guymer RH. The role of sub-retinal fluid in determining treatment outcomes in patients with neovascular age-related macular degeneration-a phase IV randomised clinical trial with ranibizumab: the FLUID study. BMC Ophthalmol. 2016;16:31

18. Keane PA, Patel PJ, Liakopoulos S, Heussen FM, Sadda SR, Tufail A. Evaluation of age-related macular degeneration with optical coherence tomography. Surv Ophthalmol. 2012;57(5):389-414.

19. Singh RP. ASRS Global trends in retina. 2018. Accessed May 5, 2021. https://www.asrs.org/content/ documents/2018-global-trends-in-retinasurvey-highlights-website.pdf

20. Brown M, Brown G, Brown H, Peet J. A value-based medicine analysis of ranibizumab for the treatment of subfoveal neovascular macular degeneration. Ophthalmology. 2008;115:1039-45.

21. Heier JS, Brown DM, Chong V, et al, for the VIEW 1 and VIEW 2 Study Groups. Intravitreal aflibercept (VEGF trap-eye) in wet age-related macular degeneration. Ophthalmology. 2012;119(12):2537-48.

22. Rosenfeld PJ, Brown DM, Heier JS, et al. Ranibizumab for neovascular agerelated macular degeneration. $\mathrm{N}$ Eng J Med. 2006;355(14):1419-31. 
23. Schmidt-Erfurth U, Chong V, Loewenstein A, et al, for the European Society of Retina Specialists. Guidelines for the management of neovascular age-related macular degeneration by the European Society of Retina Specialists (EURETINA). Br J Opthalmol. 2014;98(9):1144-67.

24. Eylea. Prescribing information. Regeneron Pharmaceuticals, Inc.; March 2021. Accessed April 21, 2021. https:// www.regeneron.com/downloads/eylea fpi.pdf

25. Lucentis. Prescribing information. Genentech, Inc.; March 2018. Accessed April 21, 2021. https://www.gene.com/ download/pdf/lucentis prescribing.pdf

26. Chong V. Ranibizumab for the treatment of wet AMD: a summary of real-world studies. Eye (Lond). 2016;30(2):270-86.

27. Gillies MC, Campain A, Barthelmes D, et al, for the Fight Retinal Blindness Study Group. Long-term outcomes of treatment of neovascular age-related macular degeneration: data from an observational study. Ophthalmology. 2015;122(9):1837-45.

28. Kataja M, Hujanen P, Huhtala H, Kaarniranta K, Tuulonen A, UusitaloJarvinen $\mathrm{H}$. Outcome of anti-vascular endothelial growth factor therapy for neovascular age-related macular degeneration in real-life setting. $\mathrm{Br} \mathrm{J}$ Ophthalmol. 2018;102(7):959-65.

29. Kiss S, Campbell J, Almony A, Shih V, Serbin M. Real-world neovascular agerelated macular degeneration treatment and outcomes: analyses of US electronic health records. Poster presented at: the American Academy of Ophthalmology (AAO) Annual Meeting; October 27-30, 2018; Chicago, IL.

30. Beovu. Prescribing information. Novartis Pharmaceuticals Corporation; October 2019. Accessed April 21, 2021. https://www.accessdata.fda.gov/drugsatfda_docs/label/2019/761125s000lbl. pdf

31. Wang P, Wang J, Ma J, Jin G, Guan X. The association between age-related macular degeneration and the risk of mortality. Biomed Res Int. 2017;3489603. doi: 10.1155/2017/3489603

32. IBM Watson Health. IBM Micromedex RED BOOK. Accessed October 2018. www.micromedexsolutions.com

33. Centers for Medicare \& Medicaid Services. Physician fee schedule search: CPT 67028. Accessed May 5, 2021. https://www.cms.gov/medicare/ physician-fee-schedule/search
34. The CATT Research Group. Ranibizumab and bevacizumab for neovascular age-related macular degeneration. N Eng J Med. 2011;364(20):1897-908.

35. Martin DF, Maguire MG, Fine SL, et al. Ranibizumab and bevacizumab for treatment of neovascular age-related macular degeneration: two-year results. Ophthalmology. 2012;119(7):1388-98.

36. Dugel PU, Jaffe GJ, Sallstig P, et al. Brolucizumab vs aflibercept in participants with neovascular age-related macular degeneration: a randomized trial. Ophthalmology. 2017;124(9):1296-304.

37. Eldem BM, Muftuoglu G, Topbaş S, et al. A randomized trial to compare the safety and efficacy of two ranibizumab dosing regimens in a Turkish cohort of patients with choroidal neovascularization secondary to AMD. Acta Ophthol. 2015;93(6):e458-e464.

38. Feltgen N, Bertelmann T, Bretag $M$, et al. Efficacy and safety of a fixed bimonthly ranibizumab treatment regimen in eyes with neovascular age-related macular degeneration: results from the RABIMO trial. Graefes Arch Clin Exp Ophthalmol. 2017;255(5):923-34.

39. Heier JS, Boyer D, Nguyen QD, et al. The 1-year results of CLEAR-IT 2, a phase 2 study of vascular endothelial growth factor trap-eye dosed as-needed after 12-week fixed dosing. Ophthalmology. 2011;118(6):1098-106.

40. Yuzawa M, Fujita K, Wittrup-Jensen KU, et al. Improvement in vision-related function with intravitreal aflibercept: data from phase 3 studies in wet age-related macular degeneration. Ophthalmology. 2015;122(3):571-78.

41. Schmidt-Erfurth U, Kaiser PK, Korobelnik JF, et al. Intravitreal aflibercept injection for neovascular age-related macular degeneration: ninety-sixweek results of the VIEW studies. Ophthalmology. 2014;121(1):193-201.

42. Ho AC, Busbee BG, Regillo CD, et al. Twenty-four-month efficacy and safety of $0.5 \mathrm{mg}$ or $2.0 \mathrm{mg}$ ranibizumab in patients with subfoveal neovascular age-related macular degeneration. Ophthalmology. 2014;121(11):2181-92.

43. Regillo CD, Brown DM, Abraham P, et al. Randomized, double-masked, sham-controlled trial of ranibizumab for neovascular age-related macular degeneration: PIER study year 1 . Am J Ophthalmol. 2008;145(2):239-48.

44. Silva R, Berta A, Larsen M, et al, for the TREND Study Group. Treat-and-extend versus monthly regimen in neovascular age-related macular degeneration. Ophthalmology. 2018;125(1):57-65.
45. Wykoff CC, Croft DE, Brown DM, et al. Prospective trial of treat-and-extend vs monthly dosing for neovascular age-related macular degeneration: TREXAMD 1-Year Results. Ophthalmology. 2015;122(12):2514-22.

46. Wykoff CC, Ou WC, Brown DM, et al. Randomized trial of treat-and-extend vs monthly dosing for neovascular age-related macular degeneration: 2-year results of the TREX-AMD study. Ophthalmology Retina. 2017;1(4):314-21.

47. Brown M, Brown G, Sharma S, Busbee B. Quality of life associated with visual loss: a time tradeoff utility analysis comparison with medical health states. Ophthalmology. 2003;110(6):1076-81.

48. Regnier S, Malcolm W, Haig J, Xue W. Cost-effectiveness of ranibizumab vs aflibercept in the treatment of visual impairment due to diabetic macular edema: a UK healthcare perspective. Clinicoecon Outcomes Res. 2015;7:235-47.

49. Institute for Clinical and Economic Review. Overview of the ICER value assessment framework and updated for 2017-2019. Accessed May 5, 2021. https:// icer.org/wp-content/uploads/2020/10/ ICER-value-assessment-frameworkUpdated-050818.pdf

50. Brecher R, Rosenfeld P, Babish D, Caplan S. Pharmacotherapy for neovascular age-related macular degeneration: an analysis of the $100 \% 2008$ Medicare fee-for-service part B claims file. Am J Ophthalmol. 2011;151(5):887-95.

51. Hernandez L, Lanitis T, Cele C, et al. Intravitreal aflibercept versus ranibizumab for wet age-related macular degeneration: a cost-effectiveness analysis. J Manag Care Spec Pharm. 2018;24(7):608-16. doi: 10.18553/ jmcp.2018.24.7.608

52. Ferreira A, Sagkriotis A, Olson M, Lu J, Makin C, Milnes F. Treatment frequency and dosing interval of ranibizumab and aflibercept for neovascular agerelated macular degeneration in routine clinical practice in the USA. PLOS ONE. 2015;10(7):e0133968.

53. Chavan R, Panneerselvam S, Adhana P, et al. Bilateral visual outcomes and service utilization of patients treated for 3 years with ranibizumab for neovascular age-related macular degeneration. Clin Ophthalmol. 2014;8:717-23. 